\title{
Multicomponent Diffusion in Crowded Solutions. 2. Mutual Diffusion in the Ternary System Tetra(ethylene glycol)-NaCl-Water
}

\author{
Alessandro Vergara, ${ }^{\dagger}$ Onofrio Annunziata, ${ }^{\ddagger}$ Luigi Paduano, ${ }^{\dagger}$ Donald G. Miller, ${ }^{\S}$ \\ John G. Albright, ${ }^{\perp}$ and Roberto Sartorio* ${ }^{*} \dagger$ \\ Dipartimento di Chimica, Università di Napoli "Federico II", Monte S. Angelo, \\ Via Cinthia, 80126 Napoli, Italy, Center for Materials Science and Engineering, Massachusetts \\ Institute of Technology, Cambridge, Massachusetts 02139, Lawrence Livermore National Laboratory, \\ Livermore, California 94551, and Department of Chemistry, Texas Christian University, \\ Fort Worth, Texas 76129
}

Received: July 29, 2003; In Final Form: October 20, 2003

\begin{abstract}
Multicomponent diffusion properties for the tetra(ethylene glycol) (PEG4)-sodium chloride-water ternary system are investigated at constant salt concentration $\left(0.5 \mathrm{~mol} \mathrm{dm}^{-3}\right)$ and a wide range of PEG4 concentration approaching $3.0 \mathrm{~mol} \mathrm{dm}^{-3}$ (60\% volume fraction). Cross-velocity correlation (counterflows) for any pair of components in this ternary system is determined. Comparison between the exact multicomponent approach and the pseudobinary approximation, which is based on the assumption that the PEG4-water mixed solvent can be treated as one component, shows how the latter approach is misleading in describing the electrolyte diffusion properties in mixed solvents. Our results show strong coupling between the fluxes of $\mathrm{NaCl}$ and PEG4, which is attributed to the $\mathrm{NaCl}-\mathrm{PEG} 4$ nonpreferential interactions in water. Moreover, the behavior of the $\mathrm{NaCl}$ and PEG4 chemical potentials as a function of the system composition can be quantitatively extracted from our diffusion measurements with the help of some interesting observations about the viscosity and conductivity properties of the system.
\end{abstract}

\section{Introduction}

The analysis of diffusion properties in aqueous solutions containing proteins and their precipitants has received increasing attention in recent years. ${ }^{1-5}$ These studies have been motivated by international programs to use microgravity conditions to grow crystals of proteins suitable for structure determination, which might not otherwise be grown under earth's gravity. In microgravity diffusion is the dominant transport mechanism in the liquid phase. ${ }^{6}$ In contrast convection may dominate in earth's gravity.

Precipitants used for protein nucleation and growth can be divided into three classes: inorganic salts, organic solvents, and polymers. While the mechanism of inorganic salts in the protein crystallization process can be reasonably described, ${ }^{7}$ the effect of polymers on the protein crystallization mechanism seems to display more complications. Since inorganic salts and polymers are often used together in the crystallization protocols, ${ }^{8}$ an analysis of such systems is a necessary step for understanding the combined effect of salts and polymers.

Among polymeric precipitants, poly(ethylene glycol), PEG, is the most widely used. PEG is a nonionic polymer that is very soluble in water, ${ }^{9}$ due to its molecular structure. Only weak nonbonding PEG-PEG interactions are present in aqueous solutions, and these interactions can be neglected in dilute conditions. In concentrated solutions, the presence of a network of PEG molecules, mediated by water bridges, has been

* Address correspondence to this author.

$\dagger$ Università di Napoli "Federico II".

Massachusetts Institute of Technology.

$\S$ Lawrence Livermore National Laboratory.

$\perp$ Texas Christian University. suggested. ${ }^{10}$ Since no direct PEG-protein interactions have been observed, the main effect of PEG on protein precipitation seems to be related to a crowding effect ${ }^{11}$ that excludes the PEG from the protein domain, thus indirectly generating an attractive force between the protein molecules.

Another important factor worth considering is the large decrease of the dielectric constant observed in aqueous PEG solutions when the polymer concentration is increased. ${ }^{5} \mathrm{~A}$ change in the dielectric constant may significantly change the activities of the ions and thus can be expected to modify the effectiveness of simple salts on the precipitation of charged proteins.

The analysis of the ternary systems PEG-salt-water is a preliminary step to understanding the properties of the quaternary system protein-PEG-salt-water, representative of crystallization protocols. PEG-salt-water systems are extensively described in the literature especially for their interesting thermodynamic properties. In fact, several systems display liquid-liquid phase separation, and the knowledge of the phase diagram is relevant for many technological applications. ${ }^{12}$ However, the characterization of their transport properties such as diffusion is not well documented.

The mutual diffusion of aqueous binary and ternary systems containing simple electrolytes is widely described in the literature, ${ }^{13}$ and predictive equations that take into account the electrostatic ion-ion interactions have been proposed and tested. The Nernst-Hartley equation is rigorously valid at infinite dilution of salt mixtures with the assumption that the solvent does not dissociate into ions and is useful for very dilute salt mixtures. ${ }^{14}$ Extensions include the Onsager-Fuoss theory, ${ }^{15}$ which accounts for the electrophoretic effect of electrolyte diffusion in binary systems, and the Pikal theory, ${ }^{16}$ which 
accounts for the effect of ion-pair formation on the electrolyte. A semiempirical correlation based on binary properties and Irreversible Thermodynamics has been effective. ${ }^{17}$

Diffusion experiments also have been performed on solutions that contain weak electrolytes ${ }^{18}$ and polyelectrolytes ${ }^{19}$ and interpreted in terms of both the mobilities of the constituents and the association constants. However, to the best of our knowledge, there is only one paper that reports diffusion coefficients of an electrolyte in a nonaqueous solvent (acetonitrile), ${ }^{20}$ and a few papers that report pseudobinary diffusion coefficients in methanol-water ${ }^{21}$ and dioxane-water mixtures. ${ }^{22}$ However, these latter systems are in fact ternary systems and require four diffusion coefficients for their correct description.

Fick's first law for a system with $n$ components and thus $n$ -1 solutes may be written:

$$
J_{i}=-\sum_{j=1}^{n-1} D_{i j} \nabla C_{j} \quad i=1, \ldots, n-1
$$

For each component $i$, there is one main-term diffusion coefficient $D_{i i}$ that gives its flow contribution due to its own concentration gradient, and $n-2$ cross-term diffusion coefficients $D_{i j}(\mathrm{i} \neq \mathrm{j})$ that give the contribution to the flow of $i$ due to the concentration gradient of each of the other solute components.

Cussler and Breuer were the first to consider a multicomponent approach for ternary aqueous mixed-solvent solutions containing salts. ${ }^{23}$ They found an extremely large cross-term diffusion coefficient for the flow of the salts due to the gradient of the organic cosolvents such as acetone and acetonitrile. They qualitatively related this large diffusion coupling to the increased thermodynamic activity of salt in the presence of the organic cosolvent. Although they noted the correlation between multicomponent diffusion and the thermodynamic behavior of the system, no microscopic interpretation of their results was reported.

Recently some of us measured the four diffusion coefficients of the ternary system PEG 400- $\mathrm{NaCl}-$ water $^{24}$ (Part 1). These measurements were made at a fixed $\mathrm{NaCl}$ concentration $(0.776$ mol dm${ }^{-3}$ ) and PEG 400 concentrations that ranged from 0.125 to $0.390 \mathrm{~mol} \mathrm{dm}^{-3}$. At the higher PEG 400 concentrations, the volume fraction was as high as $17 \%$. The effect of PEG 400 concentration on the diffusion coefficients of $\mathrm{NaCl}$ was discussed.

In this paper we report precise measurements of the diffusion coefficients $D_{i j}$ for the ternary system tetra(ethylene glycol) (PEG4) - $\mathrm{NaCl}$-water at $\mathrm{NaCl} 0.5 \mathrm{~mol} \mathrm{dm}^{-3}$, over a very wide range of PEG4 concentration (at the highest concentration the volume fraction of the PEG4 is about 60\%). We also report viscosity and volumetric measurements for the same systems because they are essential for the interpretation of the diffusion properties.

The water component is the expected choice of solvent at the lower PEG4 concentrations while at the highest concentration PEG4 becomes a logical choice of solvent. For this reason, we will report diffusion coefficients based on PEG4 as the solvent, as well as the diffusion coefficients based on water as the solvent. We will also show how ternary diffusion coefficients can be a very useful and powerful tool in describing the thermodynamic behavior of the system. Specifically, we use our diffusion results to quantitatively describe the dependence of solute chemical potentials as a function of system composition. Moreover we also provide a molecular interpretation based on excluded volume effects (hard core intermolecular potential) that is consistent with the behavior of the four diffusion coefficients.

\section{Experimental Section}

Materials. Tetra(ethylene glycol) (PEG4) was purchased from Aldrich (purity listed as 99\%+) and used without further purification. Its molar mass was taken to be $194.23 \mathrm{~g} \mathrm{~mol}^{-1}$. Sodium chloride was purchased from Aldrich (purity listed as $99.9 \%$ ), dried by heating at $450{ }^{\circ} \mathrm{C}$, taking into consideration the work of Rard, ${ }^{25}$ and used without further purification. Its molar mass was taken to be $58.443 \mathrm{~g} \mathrm{~mol}^{-1}$ and its crystal density as $2.165 \mathrm{~g} \mathrm{~cm}^{-3}$ for buoyancy corrections. ${ }^{26}$ Deionized water was distilled and then passed through a four-stage Millipore filter system to provide high-purity water for all the experiments. The molar mass of water was taken to be 18.015 $\mathrm{g} \mathrm{mol}^{-1}$.

Preparation of Solutions. Because PEG4 is hygroscopic, we prepared stock PEG4-water solutions by weight, and used them for the preparation of the ternary solutions. The densities of these stock solutions were in good agreement with the values previously determined. ${ }^{27}$ All solutions for diffusion and viscosities were prepared by weight, using $\mathrm{NaCl}$ and the $\mathrm{PEG}-$ water stock solutions with appropriate buoyancy factors. All weights were performed with a Mettler Toledo AT400 electrobalance.

Density Measurements. All density measurements were performed with a Mettler-Paar DMA40 density meter, thermostated with water from a large, well-regulated $\left( \pm 0.01{ }^{\circ} \mathrm{C}\right)$ water bath. This instrument is interfaced to a computer for time averaging, and gives precision of $\pm 2 \times 10^{-5} \mathrm{~g} \mathrm{~cm}^{-3}$ or better.

Viscosity Measurements. The viscosity measurements reported here were performed in part at Texas Christian University (ternary system $\mathrm{PEG} 4-\mathrm{NaCl}-\mathrm{H}_{2} \mathrm{O}$ ) and in part at the "Federico II" University of Naples (binary system PEG4- $\mathrm{H}_{2} \mathrm{O}$ ). In both cases an Ubbelhode viscometer in a bath at $25 \pm 0.01{ }^{\circ} \mathrm{C}$ and double distilled water as a reference liquid were used

Free-Diffusion Measurements. ${ }^{29,30}$ The diffusion experiments were performed with the Gosting diffusiometer ${ }^{30}$ operating in the Rayleigh mode. ${ }^{31,32}$ In the interferometric Rayleigh technique (as well as for the Gouy) two solutions differing slightly in concentration and initially separated by a sharp boundary are allowed to diffuse into each other. The diffusion coefficients in such experiments are obtained from the position, evolution in time, and total number of fringes $(J)$ produced by light passing through a cell containing the two solutions. Details on the experimental procedure and data analysis to extract the diffusion coefficients from the position of the interferometric fringes are reported in the Appendix.

\section{Results}

Density Data. The ternary density data have been analyzed according to the equation:

$$
d\left(c_{1}, c_{2}\right)=d\left(\bar{c}_{1}, \bar{c}_{2}\right)+H_{1}\left(c_{1}-\bar{c}_{1}\right)+H_{2}\left(c_{2}-\bar{c}_{2}\right)
$$

where $\bar{c}_{1}$ and $\bar{c}_{2}$ are the averages of the mean concentrations for all the experiments in a given series, and the $H_{i}$ are defined as:

$$
H_{i}=\left(\frac{\partial d}{\partial c_{i}}\right)_{\bar{c}_{i}, \bar{c}_{j}, i \neq j}
$$

The $d\left(\bar{c}_{1}, \bar{c}_{2}\right), H_{1}$, and $H_{2}$ are least-squares parameters, and their values are reported in Table 1 for the five compositions. 
TABLE 1: Volumetric Data for the Ternary System PEG4-NaCl-Water

\begin{tabular}{cccccccc}
\hline $\begin{array}{c}c_{1} \\
\left(\mathrm{~mol} \mathrm{dm}^{-3}\right)\end{array}$ & $\begin{array}{c}c_{2} \\
\left(\mathrm{~mol} \mathrm{dm}^{-3}\right)\end{array}$ & $\begin{array}{c}\rho \\
\left(\mathrm{g} \mathrm{cm}^{-3}\right)\end{array}$ & $\begin{array}{c}H_{1} \\
\left(\mathrm{~kg} \mathrm{~mol}^{-1}\right)\end{array}$ & $\begin{array}{c}H_{2} \\
\left(\mathrm{~kg} \mathrm{~mol}^{-1}\right)\end{array}$ & $\begin{array}{c}\bar{V}_{1} \\
\left(\mathrm{~cm}^{3} \mathrm{~mol}^{-1}\right)\end{array}$ & $\begin{array}{c}\bar{V}_{2} \\
\left(\mathrm{~cm}^{3} \mathrm{~mol}^{-1}\right)\end{array}$ & $\begin{array}{c}\bar{V}_{0} \\
\left(\mathrm{~cm}^{3} \mathrm{~mol}^{-1}\right)\end{array}$ \\
\hline 0.2500 & 0.4997 & $1.024365 \pm 0.000007$ & $0.0278 \pm 0.0005$ & $0.0394 \pm 0.0002$ & $166.8 \pm 0.5$ & $19.1 \pm 0.2$ & $18.057 \pm 0.004$ \\
0.5000 & 0.4997 & $1.031505 \pm 0.000004$ & $0.0286 \pm 0.0002$ & $0.0394 \pm 0.0001$ & $166.0 \pm 0.2$ & $19.1 \pm 0.1$ & $18.061 \pm 0.003$ \\
1.0000 & 0.5000 & $1.045986 \pm 0.000005$ & $0.0286 \pm 0.0003$ & $0.0390 \pm 0.0001$ & $166.0 \pm 0.3$ & $19.5 \pm 0.1$ & $18.054 \pm 0.007$ \\
2.0000 & 0.5000 & $1.074692 \pm 0.000003$ & $0.0287 \pm 0.0001$ & $0.0374 \pm 0.0001$ & $165.8 \pm 0.1$ & $21.1 \pm 0.1$ & $18.041 \pm 0.005$ \\
2.9921 & 0.4988 & $1.100573 \pm 0.000003$ & $0.0236 \pm 0.0002$ & $0.0359 \pm 0.0001$ & $168.6 \pm 0.1$ & $22.3 \pm 0.1$ & $17.801 \pm 0.016$
\end{tabular}

TABLE 2: Diffusion and Viscosimetric Data for the System PEG4(1)-NaCl(2)-Water ${ }^{a}$

\begin{tabular}{|c|c|c|c|c|c|}
\hline & $\mathrm{A}$ & $\mathrm{B}$ & $\mathrm{C}$ & $\mathrm{D}$ & $\mathrm{E}$ \\
\hline$c_{1}$ & 0.2500 & 0.5000 & 1.0000 & 2.0000 & 2.9921 \\
\hline$c_{2}$ & 0.4997 & 0.4997 & 0.5000 & 0.5000 & 0.4988 \\
\hline$\eta / \eta_{0}$ & 1.2172 & 1.4258 & 2.0133 & 4.2178 & 9.3664 \\
\hline$D_{11}^{0}$ & $0.6032 \pm 0.0010$ & $0.5737 \pm 0.0014$ & $0.5165 \pm 0.0004$ & $0.4030 \pm 0.0004$ & $0.2833 \pm 0.0004$ \\
\hline$D_{12}^{0}$ & $0.0371 \pm 0.0006$ & $0.0668 \pm 0.008$ & $0.1090 \pm 0.0006$ & $0.1251 \pm 0.0003$ & $0.0932 \pm 0.0006$ \\
\hline$D_{21}^{0}$ & $0.166 \pm 0.004$ & $0.157 \pm 0.005$ & $0.135 \pm 0.002$ & $0.081 \pm 0.002$ & $0.0455 \pm 0.0016$ \\
\hline$D_{22}^{0}$ & $1.311 \pm 0.002$ & $1.163 \pm 0.0025$ & $0.8940 \pm 0.0008$ & $0.488 \pm 0.006$ & $0.2316 \pm 0.0006$ \\
\hline$D_{00}^{1}$ & $0.6221 \pm 0.0026$ & $0.5917 \pm 0.0032$ & $0.5324 \pm 0.0013$ & $0.4133 \pm 0.0063$ & $0.2904 \pm 0.0023$ \\
\hline$D_{02}^{1}$ & $-1.066 \pm 0.014$ & $-1.224 \pm 0.017$ & $-1.392 \pm 0.010$ & $-1.237 \pm 0.012$ & $-0.816 \pm 0.017$ \\
\hline$D_{20}^{1}$ & $-0.0180 \pm 0.0004$ & $-0.0170 \pm 0.0005$ & $-0.0147 \pm 0.0002$ & $-0.0088 \pm 0.0002$ & $-0.0051 \pm 0.0005$ \\
\hline$D_{22}^{1}$ & $1.2921 \pm 0.0026$ & $1.1450 \pm 0.0032$ & $0.8781 \pm 0.0013$ & $0.4777 \pm 0.0063$ & $0.2243 \pm 0.0023$ \\
\hline$D_{11}^{22}$ & $0.2776 \pm 0.0085$ & $-0.007 \pm 0.013$ & $-0.412 \pm 0.011$ & $-0.5811 \pm 0.0062$ & $-0.415 \pm 0.017$ \\
\hline$D_{10}^{2}$ & $-0.0353 \pm 0.0006$ & $-0.0629 \pm 0.0008$ & $-0.1010 \pm 0.0006$ & $-0.1071 \pm 0.0003$ & $-0.0740 \pm 0.0008$ \\
\hline$D_{01}^{2}$ & $9.36 \pm 0.12$ & $10.65 \pm 0.17$ & $11.85 \pm 0.12$ & $9.73 \pm 0.13$ & $6.04 \pm 0.21$ \\
\hline$D_{00}^{2}$ & $1.6366 \pm 0.0069$ & $1.7441 \pm 0.0097$ & $1.8220 \pm 0.0065$ & $1.4721 \pm 0.0036$ & $0.9274 \pm 0.0088$ \\
\hline$T_{1}^{0}$ & 1.9142 & 1.7367 & 1.4105 & 0.8910 & 0.5149 \\
\hline$T_{1}^{1}$ & 1.9142 & 1.7367 & 1.4105 & 0.8910 & 0.5147 \\
\hline$T_{1}^{\frac{1}{2}}$ & 1.9142 & 1.7371 & 1.4100 & 0.8910 & 0.5124 \\
\hline$T_{2}^{0}$ & 0.7846 & 0.6567 & 0.4470 & 0.1865 & 0.0610 \\
\hline$T_{2}^{1}$ & 0.7846 & 0.6567 & 0.4470 & 0.1865 & 0.0610 \\
\hline$T_{2}^{2}$ & 0.7847 & 0.6577 & 0.4462 & 0.1867 & 0.0621 \\
\hline
\end{tabular}

${ }^{a}$ The molar concentrations are expressed in $\mathrm{mol} \mathrm{dm} \mathrm{dm}^{-3}$ and the diffusion coefficient in $10^{9} \mathrm{~m}^{2} \mathrm{~s}^{-1}$.

With use of the $d\left(\bar{c}_{1}, \bar{c}_{2}\right), H_{1}$, and $H_{2}$ values, the partial molar volumes of solutes 1 and 2 have been computed through the Dunlop-Gosting equation ${ }^{33}$

$$
\bar{V}_{i}=\frac{(\mathrm{MW})_{i}-1000 d}{d-c_{1} H_{1}-c_{2} H_{2}}
$$

The partial molar volume of solvent, component 0 , has been computed from the $\bar{V}_{i}$ of the two solutes by the equation:

$$
\bar{V}_{0}=\frac{1000-c_{1} \bar{V}_{1}-c_{2} \bar{V}_{2}}{c_{0}}
$$

Viscosity Data. The relative viscosities of the binary system PEG4(1) $-\mathrm{H}_{2} \mathrm{O}(0)$ were reported elsewhere. ${ }^{34}$ The following equation was obtained from the experimental data by the leastsquares method

$$
\begin{aligned}
\frac{\eta_{1}}{\eta_{0}}=1.000+ & (0.499 \pm 0.014) c_{1}+(0.494 \pm 0.020) c_{1}{ }^{2}- \\
& (0.1475 \pm 0.0081) c_{1}^{3}+(0.0666 \pm 0.0010) c_{1}{ }^{4}
\end{aligned}
$$

The viscosity data relative to the ternary system PEG4(1)$\mathrm{NaCl}(2)-\mathrm{H}_{2} \mathrm{O}(0)$, with $\left(c_{2}\right)_{\mathrm{av}}=0.4998 \pm 0.0008 \mathrm{~mol} \mathrm{dm}^{-3}$, are reported in Table 2 . Thus $\left(c_{2}\right)_{\text {av }}$ denotes the average of the salt mean concentrations for all the diffusion experiments in all the series. The following equation was obtained from the experimental data by the least-squares method

$$
\begin{array}{r}
\frac{\eta_{12}}{\eta_{0}}=1.047+(0.809 \pm 0.006) c_{1}-(0.149 \pm 0.007) c_{1}{ }^{2}+ \\
(0.270 \pm 0.002) c_{1}{ }^{3}
\end{array}
$$

where the value 1.047 at $c_{1}=0$ corresponds to the viscosity ratio $\eta_{2} / \eta_{0}$ of the binary system $\mathrm{NaCl}-\mathrm{H}_{2} \mathrm{O}$ at the average concentration $\left(c_{2}\right)_{\mathrm{av}}=0.4998 \mathrm{~mol} \mathrm{dm}^{-3}$. It was obtained by the equation ${ }^{35}$

$$
\frac{\eta_{2}}{\eta_{0}}=1.000+0.0067 c_{2}{ }^{1 / 2}+0.07866 c_{2}+0.01291 c_{2}{ }^{2}
$$

Diffusion Data. The $D_{i j}$ are reported in Table 2. The limiting value of $D_{11}$ at $c_{1}=0.0 \mathrm{~mol} \mathrm{dm}-3$ is $D_{11}^{\infty}\left(10^{9} \mathrm{~m}^{2} \mathrm{~s}^{-1}\right)=0.6324$ \pm 0.0016 and corresponds to the trace diffusion coefficients of PEG4 at infinite dilution in a solution of $\mathrm{NaCl}$ at the concentration $\left(c_{2}\right)_{\mathrm{av}}=0.4998 \pm 0.0008 \mathrm{~mol} \mathrm{dm}^{-3}$. The limiting diffusion coefficient of PEG4 in aqueous solution corresponds to $D_{1}^{\infty}\left(10^{9}\right.$ $\left.\mathrm{m}^{2} \mathrm{~s}^{-1}\right)=0.669 \pm 0.003 .{ }^{36}$ The value of $D_{22}$ at $c_{1}=0.0 \mathrm{~mol}$ $\mathrm{dm}^{-3}$ corresponds to the diffusion coefficient of the binary system $\mathrm{NaCl}(2)-\mathrm{H}_{2} \mathrm{O}(0)$ at $\left(c_{2}\right)_{\mathrm{av}}=0.4998 \pm 0.0008 \mathrm{~mol} \mathrm{dm}^{-3}$. It has been computed from the equation ${ }^{35}$

$$
\begin{aligned}
D_{2}\left(10^{9} \mathrm{~m}^{2} \mathrm{~s}^{-1}\right)=1.597-0.651 c_{2}{ }^{1 / 2}+ & 1.205 c_{2}- \\
& 0.940 c_{2}^{3 / 2}+0.272 c_{2}{ }^{2}
\end{aligned}
$$

and gives $D_{22}^{\infty}\left(10^{9} \mathrm{~m}^{2} \mathrm{~s}^{-1}\right)=1.4748$.

The cross-diffusion coefficient $D_{12}$, relative to the motion of PEG4 due to the concentration gradient of $\mathrm{NaCl}$, is zero at $c_{1}$ $=0.0 \mathrm{~mol} \mathrm{dm} \mathrm{dm}^{-3}$ by definition. However, cross-diffusion 


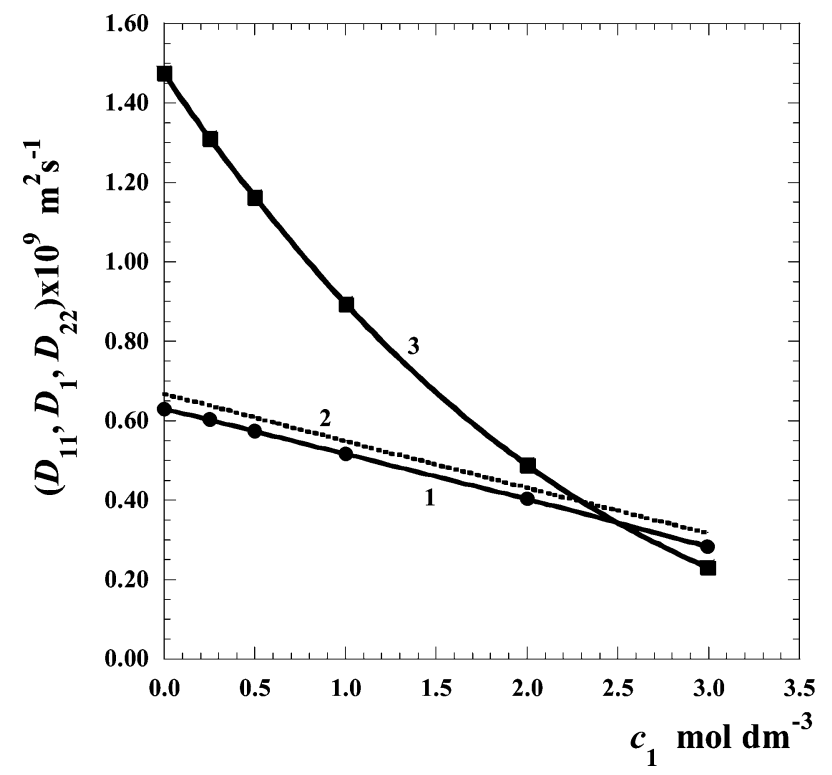

Figure 1. Main- term diffusion coefficients as a function of $c_{1}$ : curve $1, D_{11}$, and curve $2, D_{1}$; (匚) curve $3, D_{22}$.

coefficient $D_{21}$, relative to the motion of $\mathrm{NaCl}$ due to the concentration gradient of PEG4, has a finite value at $c_{1}=0.0$ $\mathrm{mol} \mathrm{dm}{ }^{-3}$ and is $D_{21}^{\infty}\left(10^{9} \mathrm{~m}^{2} \mathrm{~s}^{-1}\right)=0.226$

\section{Discussion}

Volumetric Data. The partial molar volumes $\bar{V}_{1}$ and $\bar{V}_{0}$ of PEG4 and water, respectively, are almost constant in the PEG4 concentration range we explored (see Table 1). However, it is interesting to note the large increase of the partial $\bar{V}_{2}$ of $\mathrm{NaCl}$ as the PEG4 concentration increases, a change up to about $20 \%$. A large contribution to the partial molar volume of the electrolytes in aqueous solutions is due to the strong electrostriction of water molecules in the ion hydration shells. ${ }^{37}$ This contribution is negative. For multivalent ions, it is sometimes larger than the positive contribution due to intrinsic volumes of the ions, thus leading to negative values of their partial molar volumes. ${ }^{38}$ The release of water molecules from the hydration shells to the bulk should correspond to a decrease of the electrostriction effect, which causes an increase of the values of the partial molar volumes. Then the $\bar{V}_{2}$ increment due to the increase of $c_{1}$ may simply be a decrease of water molecules into the ion hydration cospheres due to adding the highly hydrophilic PEG molecules.

Diffusion Coefficients. The main-term diffusion coefficients, $D_{11}$ and $D_{22}$, are shown in Figure 1 as a function of $c_{1}$, along with the corresponding binary values for PEG4(1)- $\mathrm{H}_{2} \mathrm{O}(0)$. The values of the cross-term diffusion coefficients $D_{12}$ and $D_{21}$ as a function of $c_{1}$ are shown in Figure 2.

The diffusion coefficients obtained by the Rayleigh technique are referred to the "volume fixed reference frame"29 and are here reported as $D_{i j}$ with the customary subscript $V$ omitted. From these coefficients, the concentrations, and the partial molar volumes of all the components, it is possible to compute the diffusion coefficients in the "solvent fixed reference frame", 39 $\left(D_{i j}\right)_{0}$, using the relations

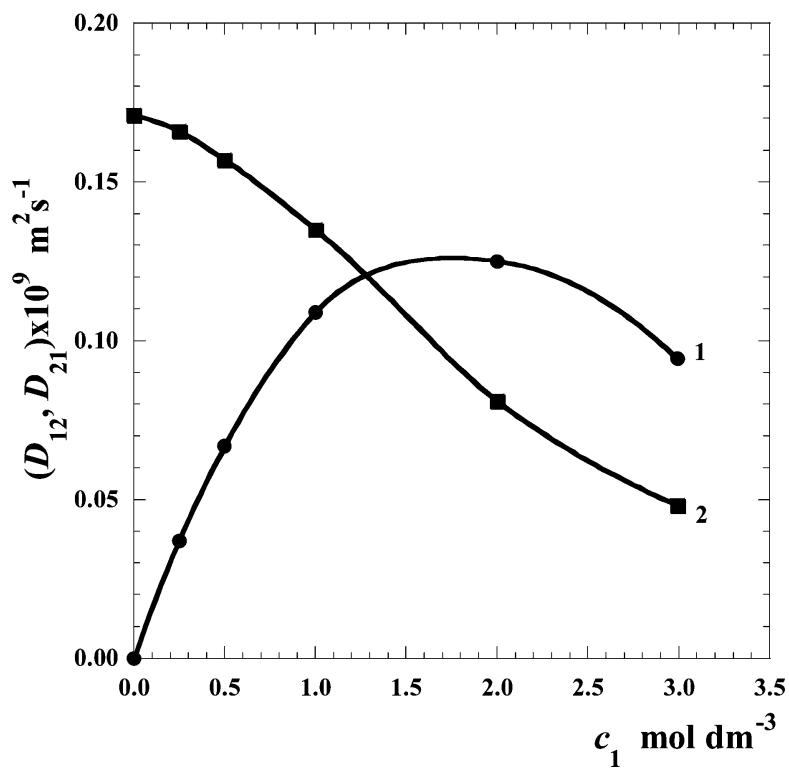

Figure 2. Cross-term diffusion coefficients as a function of $c_{1}$ : curve $1, D_{12} ;(\mathbf{\square})$ curve $2, D_{21}$.

$$
\left\{\begin{array}{l}
\left(D_{11}\right)_{0}=D_{11}+\frac{c_{1}}{c_{0} \bar{V}_{0}}\left(\bar{V}_{1} D_{11}+\bar{V}_{2} D_{21}\right) \\
\left(D_{12}\right)_{0}=D_{12}+\frac{c_{1}}{c_{0} \bar{V}_{0}}\left(\bar{V}_{1} D_{12}+\bar{V}_{2} D_{22}\right) \\
\left(D_{21}\right)_{0}=D_{21}+\frac{c_{2}}{c_{0} \bar{V}_{0}}\left(\bar{V}_{1} D_{11}+\bar{V}_{2} D_{21}\right) \\
\left(D_{22}\right)_{0}=D_{22}+\frac{c_{2}}{c_{0} \bar{V}_{0}}\left(\bar{V}_{1} D_{12}+\bar{V}_{2} D_{22}\right)
\end{array}\right.
$$

The $\left(D_{i j}\right)_{0}$ values are also a linear combination of both the ternary thermodynamic diffusion coefficients, $\left(L_{i j}\right)_{0}$ values, and the ternary thermodynamic factors $\left(\partial \mu_{i}^{T} / \partial c_{j}\right)$ denoted by $\mu_{i j}^{T}$, as shown in eq 7. According to the Onsager reciprocal relations

$$
\left\{\begin{array}{l}
\left(D_{11}\right)_{0}=\left(L_{11}\right)_{0} u_{11}^{T}+\left(L_{12}\right)_{0} \mu_{21}^{T} \\
\left(D_{12}\right)_{0}=\left(L_{11}\right)_{0} \mu_{12}^{T}+\left(L_{12}\right)_{0} \mu_{22}^{T} \\
\left(D_{21}\right)_{0}=\left(L_{21}\right)_{0} \mu_{11}^{T}+\left(L_{22}\right)_{0} \mu_{21}^{T} \\
\left(D_{22}\right)_{0}=\left(L_{21}\right)_{0} \mu_{12}^{T}+\left(L_{22}\right)_{0} \mu_{22}^{T}
\end{array}\right.
$$

(ORR), ${ }^{40}$ Onsager cross-transport coefficients are equal in the solvent-fixed frame.

Description of the Main-Term Diffusion Coefficients Behavior. $D_{11}$. The PEG4 main-term diffusion coefficient $D_{11}$, shown as curve 1 in Figure 1, is systematically smaller than the correspondent binary diffusion coefficient $D_{1}$, shown as curve 2 in the same figure. They both decrease as PEG4 concentration increases, and $D_{11}$ displays the same behavior of $D_{1}$. The $D_{11} / D_{1}$ values are constant in the concentration range $0 \leq c_{1} \leq 1.000 \mathrm{M}$, and then show a small decrease as the PEG4 concentration increases further. Moreover, the ratio $D_{11} / D_{1}$ is in excellent agreement with the ratio of the corresponding viscosities $\eta_{1} / \eta_{12}$, where $\eta_{1}$ is the viscosity of the binary system PEG4-water and $\eta_{12}$ is the viscosity of the ternary system PEG4- NaCl-water (see Table 3). These experimental observations indicate that the effect of adding $\mathrm{NaCl}$ to the PEG solutions is a hydrodynamic effect and that the thermodynamic effect can be ignored. This allowed us to conclude that the condition $\mu_{11}^{T}$ $\cong \mu_{11}^{B}$ applies. We apply the same argument to other PEG- 
TABLE 3: Prediction of $D_{11}$ and $D_{22}$

\begin{tabular}{ccccc}
\hline $\begin{array}{c}c_{1} \\
\left(\mathrm{~mol} \mathrm{dm}^{-3}\right)\end{array}$ & $\begin{array}{c}D_{11} \\
\left(10^{9} \mathrm{~m}^{2} \mathrm{~s}^{-1}\right)\end{array}$ & $\eta_{1} / \eta_{12}$ & $\begin{array}{c}D_{22} \\
\left(10^{9} \mathrm{~m}^{2} \mathrm{~s}^{-1}\right)\end{array}$ & $\eta_{2} / \eta_{12}$ \\
\hline 0.2500 & 0.6032 & 0.609 & 1.311 & 1.241 \\
0.5000 & 0.5735 & 0.579 & 1.163 & 1.066 \\
1.0000 & 0.5165 & 0.518 & 0.8940 & 0.781 \\
2.0000 & 0.4030 & 0.397 & 0.488 & 0.365 \\
2.9922 & 0.2833 & 0.281 & 0.2316 & 0.165
\end{tabular}

TABLE 4: Comparison between $\bar{V}_{1}$ and $V_{1}$

\begin{tabular}{ccc}
\hline $\begin{array}{c}c_{1} \\
\left(\mathrm{~mol} \mathrm{dm}^{-3}\right)\end{array}$ & $\begin{array}{c}\bar{V}_{1} \\
\left(\mathrm{~cm}^{3} \mathrm{~mol}^{-1}\right)\end{array}$ & $\begin{array}{c}V_{1} \\
\left(\mathrm{~cm}^{3} \mathrm{~mol}^{-1}\right)\end{array}$ \\
\hline 0.2500 & 166.81 & 307.1 \\
0.5000 & 166.04 & 303.4 \\
1.0000 & 165.98 & 291.8 \\
2.0000 & 165.76 & 254.9 \\
2.9922 & 168.57 & 231.9
\end{tabular}

$\mathrm{NaCl}$ systems and conclude that this condition is quite general for this class of systems

$D_{22}$. The $\mathrm{NaCl}$ main-term diffusion coefficient values, reported as curve 3 in Figure 1 with the corresponding $D_{2}$, decrease sharply as PEG4 molar concentration increases. At the highest PEG4 experimental concentrations, it decreases to only $15.7 \%$ of the corresponding value for the binary system $\mathrm{NaCl}$ (2) $-\mathrm{H}_{2} \mathrm{O}(0)$.

The ratio $D_{22} / D_{2}$ is significantly different from the ratio $\eta_{2} /$ $\eta_{12}$, as expected. In fact the Stokes-Einstein argument, which is based on the friction caused by a continuum medium on a macromolecule, cannot be applied to small molecules, because the hindering motion is better described by the obstruction due to quasilocalized large particles.

To investigate the PEG obstruction effect on the $\mathrm{NaCl}$ motion we have performed measurements of salt conductivity for several PEG-salt-water ternary systems, PEG2000(1)- $\mathrm{NaCl}(2)-$ water, $\mathrm{PEG} 400-\mathrm{NaCl}-$ water, and $\mathrm{PEG} 4-\mathrm{NaCl}-$ water.

In all cases, we have observed that the ratio $D_{22} / D_{2}$ is practically equal to the corresponding ratio of the ternary/binary salt conductance $\Lambda_{12} / \Lambda_{2}$. These results show that in general for all the $\mathrm{PEG}-\mathrm{NaCl}$-water systems, and in particular for the case of the PEG4- $\mathrm{NaCl}$-water ternary system, see Table 4 , as the PEG concentration increases, both $D_{22}$ and salt conductivity decrease with the same dependence on PEG concentration. The salt diffusion coefficient depends not only on the mobility of the ions but also on the thermodynamic properties of the system. This is a very important difference with respect to the conductance, which depends only on the mobility of the ions. Since conductivity and salt diffusion have the same behavior with respect to the PEG concentration, we expect that the value of $\mu_{22}$ is essentially not changed by the presence of PEG.

Calculation of the Chemical Potential Derivatives. Our significant observations on the conductivity and viscosity properties of the system allow us to take $\mu_{11}^{T}$ and $\mu_{22}^{T}$ from the available ${ }^{41,35}$ binary data. Using the values of $\mu_{11}^{T}$ and $\mu_{22}^{T}$, we can determine the cross-chemical potential derivatives, $\mu_{12}^{T}$ and $\mu_{21}^{T}$, with a procedure suggested recently by some of us. ${ }^{2}$

This procedure is based on the application of Euler's condition:

$$
\mu_{12}^{T}\left(1-C_{2} \bar{V}_{2}\right)-\mu_{11}^{T} C_{1} \bar{V}_{2}=\mu_{21}^{T}\left(1-C_{1} \bar{V}_{1}\right)-\mu_{22}^{T} C_{2} \bar{V}_{1}
$$

and the ORR:

$$
\mu_{11}^{T}\left(D_{12}\right)_{0}-\mu_{12}^{T}\left(D_{21}\right)_{0}=\mu_{22}^{T}\left(D_{21}\right)_{0}-\mu_{21}^{T}\left(D_{22}\right)_{0}
$$

TABLE 5: Derivatives of the Chemical Potentials

\begin{tabular}{ccccc}
\hline $\begin{array}{c}c_{1} \\
\left(\mathrm{~mol} \mathrm{dm}^{-3}\right)\end{array}$ & $\begin{array}{c}\mu_{11} / R T \\
\mathrm{dm}^{3} \mathrm{~mol}^{-1}\end{array}$ & $\begin{array}{c}\mu_{12} / R T \\
\mathrm{dm}^{3} \mathrm{~mol}^{-1}\end{array}$ & $\begin{array}{c}\mu_{21} / R T \\
\mathrm{dm}^{3} \mathrm{~mol}^{-1}\end{array}$ & $\begin{array}{c}\mu_{22} / R T \\
\mathrm{dm}^{3} \mathrm{~mol}^{-1}\end{array}$ \\
\hline 0.2500 & 4.556 & 0.32 & 0.63 & 3.756 \\
0.5000 & 2.574 & 0.31 & 0.65 & 3.756 \\
1.0000 & 1.618 & 0.31 & 0.70 & 3.754 \\
2.0000 & 1.225 & 0.35 & 0.90 & 3.754 \\
2.9922 & 1.206 & 0.35 & 1.17 & 3.763
\end{tabular}

TABLE 6: Thermodynamic Diffusion Coefficients

\begin{tabular}{ccccc}
\hline $\begin{array}{c}c_{1} \\
\left(\mathrm{~mol} \mathrm{dm}^{-3}\right)\end{array}$ & $\begin{array}{c}R T\left(L_{11}\right)_{0} \\
\left(10^{9} \mathrm{~m}^{2} \mathrm{~s}^{-1}\right. \\
\left.\mathrm{mol} \mathrm{dm}^{-3}\right)\end{array}$ & $\begin{array}{c}R T\left(L_{12}\right)_{0} \\
\left(10^{9} \mathrm{~m}^{2} \mathrm{~s}^{-1}\right. \\
\left.\mathrm{mol} \mathrm{dm}^{-3}\right)\end{array}$ & $\begin{array}{c}R T\left(L_{21}\right)_{0} \\
\left(10^{9} \mathrm{~m}^{2} \mathrm{~s}^{-1}\right. \\
\left.\mathrm{mol} \mathrm{dm}^{-3}\right)\end{array}$ & $\begin{array}{c}R T\left(L_{22}\right)_{0} \\
\left(10^{9} \mathrm{~m}^{2} \mathrm{~s}^{-1}\right. \\
\left.\mathrm{mol} \mathrm{dm}^{-3}\right)\end{array}$ \\
\hline 0.2500 & 0.139 & 0.000 & 0.000 & 0.354 \\
0.5000 & 0.244 & 0.003 & 0.003 & 0.314 \\
1.0000 & 0.382 & 0.009 & 0.009 & 0.243 \\
2.0000 & 0.486 & 0.013 & 0.013 & 0.132 \\
2.9922 & 0.473 & 0.015 & 0.015 & 0.066
\end{tabular}

The $\mu_{12}^{T}$ and $\mu_{21}^{T}$ values, obtained solving eqs 8 and 9 , are reported in Table 5. As we can see from the table, the $\mu_{12}^{T}$ are positive and almost independent of the PEG4 molar concentration. On the other hand the $\mu_{21}^{T}$ values, also positive, increase with the PEG4 molar concentration. The magnitude and the behavior of $\mu_{21}^{T}$, as we are going to see in the next section, can be explained in terms of excluded volume effects.

By using the $\mu_{i j}^{T}$ values it is then possible to compute the values of the $\left(L_{i j}\right)_{0}$ that are reported in Table 6. It is worth noting that the cross-thermodynamic coefficients are negligible with respect to the main-thermodynamic coefficients at least in the range $0 \leq c_{1} \leq 1.000 \mathrm{M}$. This is not completely unexpected because of the absence of strong specific PEG4- $\mathrm{NaCl}$ interaction. This absence of PEG4- $\mathrm{NaCl}$ interaction will allow us, to explicitly relate the cross-diffusion coefficients to the $\mu_{i j}^{T}$ quantities without the use of the thermodynamic diffusion coefficients.

Description of the Cross-Term Diffusion Coefficient's Behavior. Because of the values of the chemical potential derivatives and of the thermodynamic diffusion coefficients, at least in the concentration range $0 \leq c_{1} \leq 1.000 \mathrm{M}$, we can rewrite eqs 7 as

$$
\left\{\begin{array}{l}
\left(D_{11}\right)_{0} \cong\left(L_{11}\right)_{0} \mu_{11}^{T} \\
\left(D_{12}\right)_{0} \cong\left(L_{11}\right)_{0} \mu_{12}^{T} \\
\left(D_{21}\right)_{0} \cong\left(L_{22}\right)_{0} \mu_{21}^{T} \\
\left(D_{22}\right)_{0} \cong\left(L_{22}\right)_{0} \mu_{22}^{T}
\end{array}\right.
$$

For PEG4 chemical potentials we have

$$
\mu_{1}^{T}=\mu_{1}^{0}+R T \ln c_{1}+R T \beta_{1}^{T}
$$

where $R T \beta_{1}$ is the chemical potential excess. By differentiation of $\mu_{1}^{T}$, we obtain the following expressions

$$
\left\{\begin{array}{l}
\frac{\mu_{11}^{T}}{R T}=\frac{1}{c_{1}}+\beta_{11}^{T} \\
\frac{\mu_{12}^{T}}{R T}=\beta_{12}^{T}
\end{array}\right.
$$

where

$$
\beta_{i j}^{B}=\left(\frac{\partial \beta_{i}^{B}}{\partial c_{j}}\right) \quad \text { with } i, j=1,2
$$

Using eqs 10 and 12 we obtain for the $\left(D_{12}\right)_{0}$ and $\left(D_{21}\right)_{0}$ the 
following expressions

$$
\left(D_{12}\right)_{0} \cong\left(D_{11}\right)_{0} \frac{c_{1} \beta_{12}^{T}}{1+c_{1} \beta_{11}^{T}} \cong\left(D_{11}\right)_{0} \frac{c_{1} \beta_{12}^{T}}{1+c_{1} \beta_{11}^{B}}
$$

We can now analyze the experimental behavior of $\left(D_{12}\right)_{0}$ as a function of $c_{1}$ using eq 13. This diffusion coefficient, which vanishes at zero PEG4 molar concentration, becomes and remains positive at all the concentrations explored. As we can see from Figure 2, the value of $\left(D_{12}\right)_{0}$ increases up to $c_{1} \cong 1.60$ mol dm${ }^{-3}$ and then decreases when the concentration of PEG4 becomes larger.

This behavior can be deduced from eq 13. Since both $\beta_{11}^{T}$ and $\beta_{12}^{T}$ are positive and roughly constant $\left(\beta_{11}^{T}\right.$ can be estimated as $\beta_{11}^{T} \cong \mu_{11}^{B} / R T-1 / c_{1}$, and $\left.\beta_{12}^{T} \cong \mu_{12}^{T} / R T\right)$, we can see that the second factor in eq 13 increases for small values of $c_{1}$ and then becomes roughly constant at high PEG4 concentration. Thus, for small $c_{1}$, the behavior of $\left(D_{12}\right)_{0}$ as a function of $c_{1}$ is determined by the second factor while at large PEG4 concentration it reflects the decrease of $\left(D_{11}\right)_{0}$.

In the case of the salt chemical potential, we can assume that the salt concentration is increased due to the excluded volume effect of PEG4 and that the salt chemical potential in the ternary system is simply equal to the salt chemical potential in the corresponding binary system at the effective concentration $c_{2}^{*}$ where $^{42}$

$$
c_{2}^{*}=\frac{c_{2}}{1-V_{1} c_{1}}
$$

and $V_{1}$ is the "effective" salt excluded volume due to $1 \mathrm{~mol}$ of PEG.

Thus we can write:

$$
\mu_{2}^{T}\left(c_{1}, c_{2}\right)=\mu_{2}^{B}\left(c_{2}^{*}\right)=\mu_{2}^{0}+2 R T \ln c_{2}^{*}+R T \beta_{2}^{B} *
$$

where $\beta_{2}^{B^{*}}$ is the value of $\beta_{2}^{B}$ calculated at the effective salt concentration $c_{2}^{*}$. In this case we obtain

$$
\left\{\begin{array}{l}
\frac{\mu_{22}^{T}}{R T}=\frac{1}{1-c_{1} V_{1}}\left(\frac{2}{c_{2}^{*}}+\beta_{22}^{B^{*}}\right) \\
\frac{\mu_{21}^{T}}{R T}=\frac{c_{2} V_{1}}{\left(1-V_{1} c_{1}\right)^{2}}\left(\frac{2}{c_{2}^{*}}+\beta_{22}^{B^{*}}\right)
\end{array}\right.
$$

and then

$$
\left(D_{21}\right)_{0} \cong\left(D_{22}\right)_{0} \frac{V_{1} c_{2}}{\left(1-V_{1} c_{1}\right)}
$$

The cross-term diffusion coefficient $\left(D_{21}\right)_{0}$ is finite at zero PEG4 concentration and positive over the range of experiments, but decreases as the PEG4 concentration increases (see Figure 2). As we can see from eq 17 , the decrease of $\left(D_{21}\right)_{0}$ is related to the corresponding decrease of $\left(D_{22}\right)_{0}$. We can also use eq 17 to calculate the values of $V_{1}$. As we can see from Table 4 , the $V_{1}$ values are always larger than the $\bar{V}_{1}$ values, which is consistent with the presence of a PEG-water preferential interaction and corresponding PEG-salt nonpreferential interactions. ${ }^{5,23,43}$ Moreover, the difference between $V_{1}$ and $\bar{V}_{1}$ decreases as $c_{1}$ increases. This behavior is consistent with the corresponding decrease of water content inside the system. It is interesting to observe that our excluded volume values (see Table 4) are comparable with

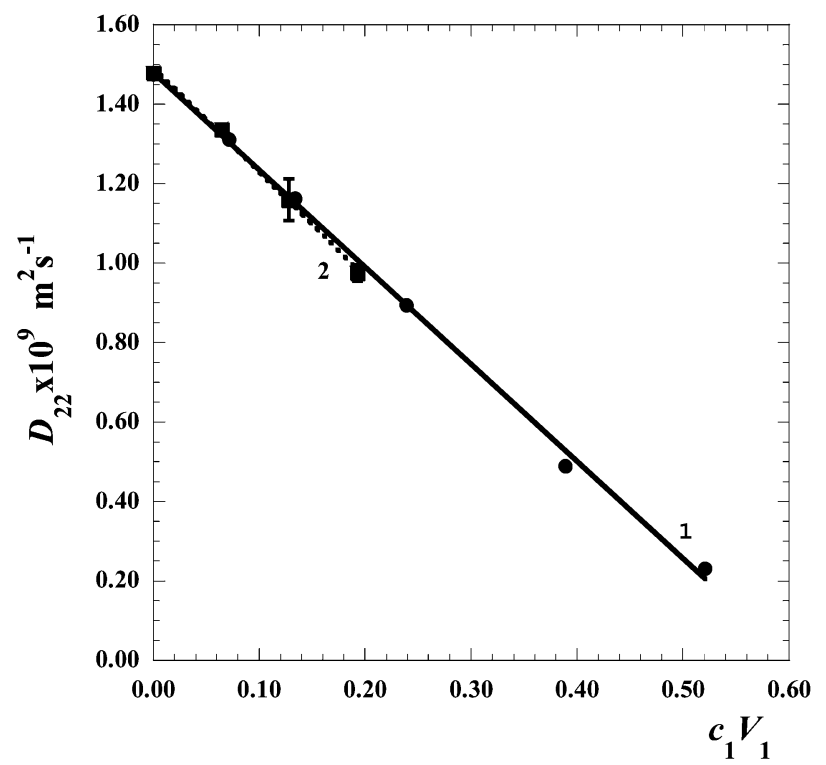

Figure 3. Main-term diffusion coefficient $D_{22}$ as a function of the PEG volume fraction $c_{1} V_{1}$ : (๑) curve 1 , system PEG4(1)- $\mathrm{NaCl}(2)-$ water, and (ם) curve 2, system PEG 400(1)- $\mathrm{NaCl}(2)$ - water.

the PEG4 hydrodynamic volume, $V_{1}=225.7 \mathrm{~cm}^{3} \mathrm{~mol}^{-1}$, obtained by diffusion analysis on the PEG4-water binary system $^{36}$

\section{Further Comments on $D_{i j}$}

Diffusion Coefficients with Different Solvent Choices. It is interesting to discuss the diffusion coefficients referred to solvent choices other than water. Note that, in this section, the solvent choice will be indicated by a superscript on the diffusion coefficients (not to be confused with the reference frame). In Table 2 we report the values of $D_{i j}^{1}$ and $D_{i j}^{2}$. These quantities can be obtained from the experimental $D_{i j}^{0}$ values and the partial molar volumes of all the components.

Since pure PEG4 is a liquid and is completely miscible with water, it is also appropriate to treat this component as solvent and examine some interesting results. Figure 3 shows the values of the diffusion coefficients $D_{02}^{1}$ relative to the motion of water under the $\mathrm{NaCl}$ concentration gradient with PEG4 as solvent. $D_{02}^{1}$ is always negative and presents a sharp minimum when reported as a function of $c_{1}$. It is interesting to analyze the limiting behavior of this diffusion coefficient as the concentration of water goes to zero. By using the ternary density data it is possible to obtain, by extrapolation, the density of the binary system $\mathrm{NaCl}-\mathrm{PEG} 4$ at the salt concentration $\left(c_{2}\right)_{\mathrm{av}}=0.4998$ $\pm 0.0008 \mathrm{~mol} \mathrm{dm}^{-3}$.

At zero water concentration, we calculate $c_{1}=5.8513 \mathrm{~mol}$ $\mathrm{dm}^{-3}$. The diffusion coefficient $D_{02}^{1}$ must approach zero when the water concentration is zero and the experimental data show this trend (see Figure 3 ). The diffusion coefficient $D_{01}^{2}$ displays a similar behavior.

It is important to remark that at $c_{1}$ larger than $0.5 \mathrm{~mol} \mathrm{dm}^{-3}$, the PEG main term $D_{11}^{2}$ is negative! This is unusual, but not prohibited. ${ }^{44}$ In fact, it can be shown that only the conditions $T_{1} \equiv D_{11}^{2}+D_{22}^{2}>0$ (trace) and $T_{2} \equiv\|D\|$ (determinant) must hold for stable diffusion, and that both $T_{1}$ and $T_{2}$ must be the same for all solvent choices, i.e., $T_{1}^{0}=T_{1}^{1}=T_{1}^{2}$ and $T_{2}^{0}=T_{2}^{1}=$ $T_{2}^{2}$. As expected, our experimental results satisfy these conditions, see Table 2 (these conditions do not hold in other reference frames if the driving forces are $\left.\partial c_{i} / \partial x\right) .{ }^{44}$ 


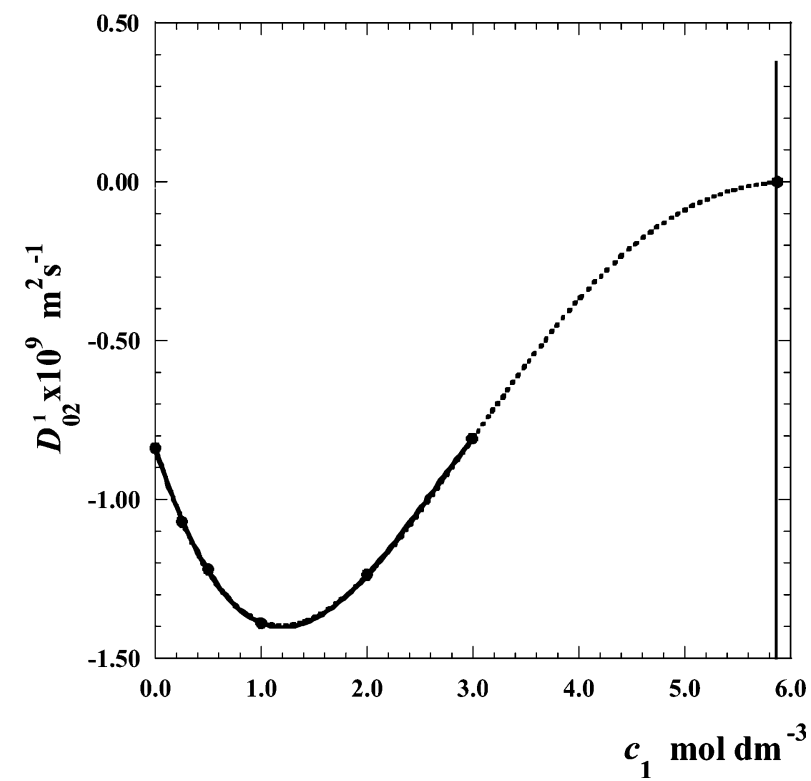

Figure 4. Cross term diffusion coefficient as a function of $c_{1}$.

Diffusion Coefficients of $\mathrm{NaCl}$ in the "Mixed Solvent" (PEG4- $\mathbf{H}_{2} \mathbf{O}$ ). An attractive but dangerous way of describing such a ternary system is to determine (experimentally) and to report pseudobinary diffusion coefficients of $\mathrm{NaCl}$ with respect to a mixed PEG-water solvent. In this last section we are going to show that the salt transport properties are inaccurately described by this misleading approach.

In an experimental scenario where only the salt transport properties are of interest, an initial gradient of $\mathrm{NaCl}$ concentration needs to be prepared. Due to both the mass balance and the fluid incompressibility, a corresponding concentration gradient of the solvent can be calculated in a binary system. In a ternary system, where two components are considered as solutes, this is not possible. However, the ratio between the concentrations of the two solvents, consistently with the pseudobinary condition, can be fixed from the ratio between their initial gradients:

$$
\frac{c_{0}}{c_{1}}=\frac{\Delta c_{0}}{\Delta c_{1}}
$$

Equation 18 allows us to determine the three concentration gradients occurring in a ternary system. The pseudobinary diffusion coefficient $D_{A}$ is defined in terms of the eigenvalues $\lambda_{1}$ and $\lambda_{2}$ of the ternary diffusion coefficient matrix ${ }^{45}$

$$
\frac{1}{\sqrt{D_{A}}}=\Gamma_{1} \frac{1}{\sqrt{\lambda_{1}}}+\Gamma_{2} \frac{1}{\sqrt{\lambda_{2}}}
$$

The weights $\Gamma_{1}$ and $\Gamma_{2}\left(\Gamma_{1}+\Gamma_{2}=1\right)^{14}$ can be easily calculated from the initial gradients (represented by the $\Delta c_{i}$ values) and the available refractive index increments,

We calculate the pseudobinary diffusion coefficients $D_{A}$. The results for $D_{A}$ obtained with eq 19 are reported in Figure 5. The difference observed between the behaviors of $D_{22}$ and $D_{A}$ as functions of $c_{1}$ are quite significant. As we can see from the figure, $D_{22}$ decreases sharply when $c_{1}$ increases. On the contrary, $D_{A}$ first increases and then decreases toward a value that is about four times larger than the corresponding $D_{22}$ value. Thus, we conclude that the pseudobinary approach qualitatively fails to describe the salt diffusion properties in the mixed solvent.

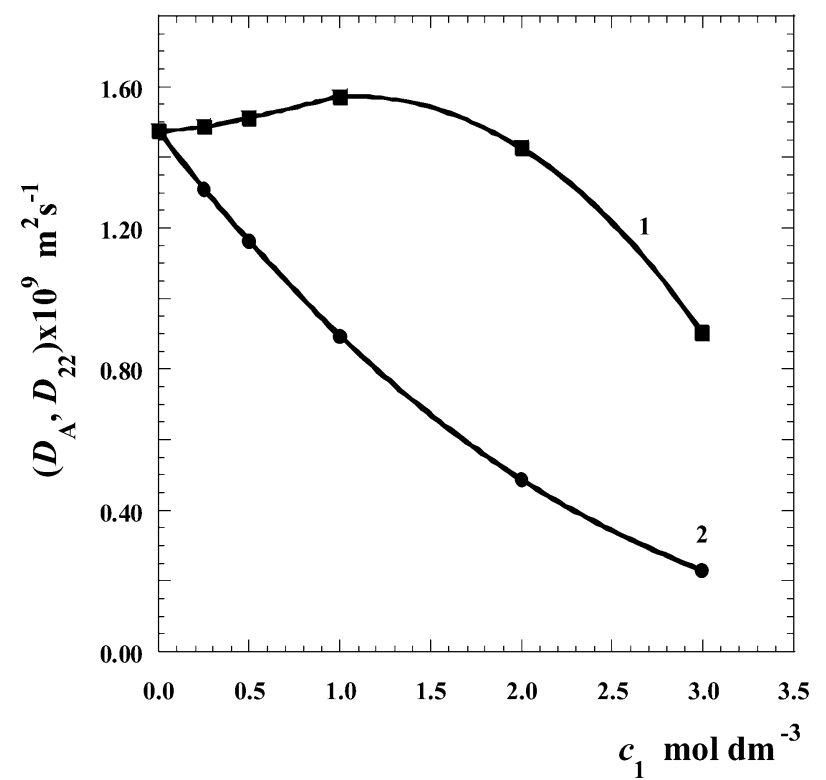

Figure 5. Main-term diffusion coefficient $D_{22}$ and $\mathrm{NaCl}$ pseudobinary diffusion coefficient $D_{A}$ as a function of $c_{1}$ : (†) curve $1, D_{22} ;(\boldsymbol{\square})$ curve $2, D_{A}$.

\section{Conclusion}

We presented the transport properties of the ternary system PEG4- $\mathrm{NaCl}-\mathrm{H}_{2} \mathrm{O}$, where both PEG4 and water can be regarded as a solvent. We have shown that the diffusion properties of an electrolyte in a mixed solvent can be properly described only in the case where the four diffusion coefficients are available, i.e., the pseudobinary approximation qualitatively fails to describe the electrolyte diffusion. Moreover, we used the four diffusion coefficients to determine the dependence of the chemical potentials as a function of solution composition. We demonstrated that the two cross-diffusion coefficients are directly related to the equilibrium properties of the system for $\mathrm{NaCl}$ in aqueous PEG solutions and interpreted them by taking into account excluded volume concepts and the presence of $\mathrm{PEG}-\mathrm{NaCl}$ nonpreferential interactions.

Acknowledgment. This research was supported by the ASI contracts ARS-99-15 and IFN/ASI ARS 99-17 and at TCU by NASA grant NAG8-1356.

\section{Appendix}

Rayleigh fringes reflect the refractive index distribution in the diffusion cell, and their positions shift as the boundary is traversed. This results from the change in refractive index with vertical position in the diffusion channel, compared to the refractive index of water or a solution of uniform composition in the reference channel. Since concentration differences in the diffusion channel are small in our experiments, a truncated Taylor series expansion of the refractive index around the mean concentration is adequate to relate refractive index to concentration, and yields for a ternary system

$$
n=\bar{n}+R_{1}\left(C_{1}-\bar{C}_{2}\right)+R_{2}\left(C_{2}-\bar{C}_{2}\right)
$$

where $n$ is the refractive index at position $\mathrm{x}, C_{i}$ is the concentration of solute $i$ at position $\mathrm{x}, C_{i}$ is the average concentration of solute $i, \bar{n}$ is the refractive index at the mean concentrations, and $R_{i}$ is the refractive index increment of solute $i$. The bar denotes mean concentrations of the given experiment. 
For the usual case where the $D_{i j}$ matrix has distinct eigenvalues, substitution of the appropriate solution of Fick's law for free diffusion boundary conditions into eq A1 yields

$$
f(i)=\left(\frac{2 j-J}{J}\right)=\left(\frac{n-\bar{n}}{\Delta n / 2}\right)=\Gamma_{1} \operatorname{erf}\left(\frac{s_{1} x_{j}}{2 t^{1 / 2}}\right)+\Gamma_{2} \operatorname{erf}\left(\frac{s_{2} x_{j}}{2 t^{1 / 2}}\right)
$$

where $f(j)$ is the reduced fringe number and $x$ is the distance from the center of the boundary and is positive downward. The difference in refractive index across the boundary, $\Delta n$, is related to $J$ by

$$
\Delta n=(J \lambda / a)=R_{1} \Delta C_{1}+R_{2} \Delta C_{2}
$$

where $\lambda$ is the wavelength of the light. The $\Gamma_{i}$ coefficients are independent of the boundary conditions, and their expressions are given below. Since the $R_{i}$ appear as ratios in subsequent equations, $\lambda / a$ in eq A3 need not be determined.

For the distinct eigenvalues case

$$
\Gamma_{1}+\Gamma_{2}=1
$$

and $\Gamma_{i}$ can be written as

$$
\Gamma_{i}=a+b \alpha_{1}
$$

where

$$
\alpha_{i}=\left(\frac{R_{i} \Delta C_{i}}{R_{1} \Delta C_{1}+R_{2} \Delta C_{2}}\right)
$$

$a$ and $b$ are the following functions of both the $D_{i j}$ and $R_{i}$

$$
\begin{gathered}
a=\left(\frac{s_{1}^{2} \mathbf{D}-D_{22}-D_{12}\left(R_{1} / R_{2}\right)}{\mathbf{D}\left(s_{1}^{2}-s_{2}^{2}\right)}\right) \\
b=\left(\frac{D_{22}+D_{12}\left(R_{1} / R_{2}\right)-D_{11}-D_{21}\left(R_{2} / R_{1}\right)}{\mathrm{D}\left(s_{1}^{2}-s_{2}^{2}\right)}\right)
\end{gathered}
$$

and $\mathbf{D}$ is the determinant of the diffusion coefficient matrix. The $s_{i}$ are functions only of the $D_{i j}$, are related to the eigenvalues $\lambda_{i}$ of the $D_{i j}$ matrix, and are given by

$$
\begin{gathered}
\lambda_{1}=\left(\frac{\left(D_{11}+D_{22}\right)-\left[\left(D_{11}+D_{22}\right)^{2}-4 \mathrm{D}\right]^{1 / 2}}{2}\right) \\
\lambda_{2}=\left(\frac{\left(D_{11}+D_{22}\right)+\left[\left(D_{11}+D_{22}\right)^{2}-4 \mathrm{D}\right]^{1 / 2}}{2}\right) \\
s_{i}=1 / \lambda_{i}^{1 / 2}
\end{gathered}
$$

From eq A2 it is seen that $f(j)=f(J-j)$ and $x_{j}=-x_{(j-j)}$. These are the two x-positions of a Creeth pair, and their difference is twice for each one. Consequently $f(j)$ and $\bar{x}_{j}$

$$
\bar{x}_{j}=\frac{x_{j}-x_{(J-j)}}{2}
$$

are sufficient to characterize Creeth pairs. Only Creeth pairs whose $j$ values lie between 0 and $J / 2$ are required, since they contain all the fringe position information. As mentioned earlier, the position of the center of the pattern is not needed for Creeth pair calculations. Note that both $f(j)$ and $\bar{x}_{j}$ are negative for all $j<J / 2$, so signs can be reversed for programming purposes.

It is convenient to define a "reduced fringe position" $y_{j}$ as

$$
y_{j}=\left(\frac{\bar{x}_{j}}{2 t^{1 / 2}}\right)
$$

This $y_{j}$ is independent of the corrected time (i.e., should be the same for all exposures) for a given $j$. For a given $j$, if there is a large deviation of $y_{j}$ in one pattern from the average $y_{j}$ of all the patterns, it signifies an error in reading a fringe position.

Consequently, that $y_{j}$ can be discarded and the remaining $y_{j}$ re-averaged. Since we have only 9 or 10 exposures, we actually use all individual values of $y_{j}$ after any such outliers are discarded.

The average $y_{j}$ is the representative position for that $j$. The set of average $y_{j}$ values for all $j$ values thus forms a representative Rayleigh pattern. Use of this representative pattern will be essential with automated data acquisition, which could yield the equivalent of 20 to 1000 exposures.

For the least-squares analysis of data, eq A2 can be rewritten as

$$
f(j)=\left(a+b \alpha_{1}\right) \operatorname{erf}\left(s_{1} y_{j}\right)+\left(1-a-b \alpha_{1}\right) \operatorname{erf}\left(s_{2} y_{j}\right)
$$

The four least-squares parameters are $a, b, s_{1}$, and $s_{2}$.

Equation A14 refers to a given fringe in a given exposure in a given experiment, for which the experimental quantities are $\left(j, x, x_{(J-j)}, t\right)$, taking into account eqs A12 and A13. The quantities $J$ and $\alpha_{1}$ are experimental quantities associated with each experiment.

Finally the quantities $a, b, s_{1}$, and $s_{2}$ and the $D_{i j}$ calculated from them are derived quantities associated with the combined set of experiments. Although $\Gamma_{1}, s_{1}$, and $s_{2}$ can be obtained by least squares from a single experiment, the $a$ and $b$ (and $R_{1}$ and $R_{2}$ as well) cannot be obtained without data from two or more experiments with different $a_{1}$ values, i.e., from two or more different $\Delta C_{1} / \Delta C_{2}$ ratios.

The four diffusion coefficients can be determined from $a, b$, $s_{1}$, and $s_{2}$ by using the equations ${ }^{46-50}$

$$
\begin{gathered}
D_{11}=\left(\frac{(a+b)(1-a) s_{2}^{2}-a(1-a-b) s_{1}^{2}}{b s_{1}^{2} s_{2}^{2}}\right) \\
D_{12}=\left(\frac{\left(-R_{2} / R_{1}\right)\left(s_{1}^{2}-s_{2}^{2}\right) a(1-a)}{b s_{1}^{2} s_{2}^{2}}\right) \\
D_{21}=\left(\frac{\left(-R_{2} / R_{1}\right)\left(s_{1}^{2}-s_{2}^{2}\right)(a+b)(1-a-b)}{b s_{1}^{2} s_{2}^{2}}\right) \\
D_{22}=\left(\frac{(a+b)(1-a) s_{2}^{2}-a(1-a-b) s_{2}^{2}}{b s_{1}^{2} s_{2}^{2}}\right)
\end{gathered}
$$

We note that if the eigenvalues of the diffusion coefficient matrix are exactly equal or nearly equal, then a different analysis will be required.

\section{References and Notes}

(1) Albright, J. G.; Annunziata, O.; Miller, D. G.; Paduano, L.; Pearlstein, J. J. Am. Chem. Soc. 1999, 121, 3256.

(2) Annunziata, O.; Paduano, L.; Pearlstein, A. J.; Miller, D. G.; Albright, J. G. J. Am. Chem. Soc. 2000, 122, 5916. 
(3) Paduano, L.; Annunziata, O.; Pearlstein, A. J.; Miller, D. G.; Albright, J. G. J. Cryst. Growth 2001, 232, 273.

(4) Izmailov, A. F.; Myerson, A. S. J. Chem. Phys. 2000, 112, 4357.

(5) Vergara, A.; Paduano, L.; Sartorio, R. Macromolecules 2002, 35 , 1389.

(6) Lin, H.; Rosenberger, F.; Alexander J. I. D.; Nadarajah, A. J. Cryst. Growth 1995, 151, 153 .

(7) Rosenbaum, D. F.; Kulkarni, A.; Ramakrishnan, S.; Zukoski, C. F. J. Phys. Chem. 1999, 111, 9882.

(8) Ray, W. J., Jr. PROTEINS: Struct., Funct., Genet. 1992, 14, 300. 2053.

(9) Kjellander, R.; Florin, E. J. Chem. Soc., Faraday Trans. 1981, 77

(10) Vergara, A.; Paduano, L.; D’Errico, G.; Sartorio, R. Phys. Chem. Chem. Phys. 1999, 1 (20), 4875.

(11) Minton, A. P. J. Biol. Chem. 2001, 276 (14), 10577.

(12) Meller da Silva, L. H.; Coimbra, J. S. R.; Meirelles, A. J. J. Chem. Eng. Data 1997, 42, 398.

(13) Miller, D. G. J. Phys. Chem. 1959, 63, 570.

(14) Gosting, L. J. Adv. Protein Chem. 1956, 11, 429.

(15) Harned, H. S.; Owen, B. B. The Physical Chemistry of Electroytic Solutions; Reinhold Publishing Corp.: New York, 1943.

(16) Pikal, M. J. J. Phys. Chem. 1971, 75, 663.

(17) Miller, D. G. J. Phys. Chem. 1967, 71, 616.

(18) Vitagliano, V.; Lyons, P. A. J. Am. Chem. Soc. 1956, 78, 4538

(19) Ambrosone, L.; Costantino, L.; Guarino, G.; Sartorio, R.; Vitagliano,

V. Gazz. Chim. Ital. 1986, 116, 625

(20) Lyons, P. A.; Renner, T. A. J. Phys. Chem. 1974, 78, 1667.

(21) Leaist, D. G.; Lyons, P. A. J. Solution Chem. 1981, 10, 95.

(22) Havenga, E.; Leaist, D. G. J. Chem. Soc., Faraday Trans. 1998, $94,3353$.

(23) Cussler, E. L.; Breuer, M. M. AIChE J. 1972, 18, 812.

(24) Vergara, A.; Paduano, L.; Vitagliano, V.; Sartorio, R. Macromolecules 2001, 34, 991 .

(25) Rard, J. A. J. Chem. Thermodyn. 1996, 28, 83.

(26) Weast, R. C. CRC Handbook of Chemistry and Physics, 557th ed.; CRC Press: Cleveland, OH, 1975.

(27) Ambrosone, L.; D’Errico, G.; Sartorio, R.; Costantino, L. J. Chem. Soc., Faraday Trans. 1997, 93 (22), 3961.
(28) Tyrrell, H. J. V.; Harris, K. R. Diffusion in liquids; Butterworths: London, UK, 1984.

(29) Miller, D. G.; Albright, J. G. In Measurements of the Transport Properties of Fluids: Experimental Thermodynamics; Wakeham, W. A. Nagashima, A., Sengers, J. V., Eds.; Blackwell Scientific Publication: Oxford, UK, 1991; p 272.

(30) Gosting, L. J.; Kim, H.; Loewenstein, M. A.; Reinfeldsa, G.; Revzin,

A. Rev. Sci. Instrum. 1973, 44, 1602.

(31) Rard, J. A.; Miller, D. G.; Albright, J. G.; Mathew, R.; Lee, L. M.; Zeidler, M. E. J. Phys. Chem. 1993, 97, 3885.

(32) Rard, J. A.; Miller, D. G.; Albright, J. G.; Lee, L. M.; Zeidler, M. E. J. Chem. Soc., Faraday Trans. 1996, 92, 4187

(33) Dunlop, P. J.; Gosting, L. J. J. Phys. Chem. 1959, 63, 86.

(34) Vergara, A.; Paduano, L.; Sartorio, R. J. Phys Chem. B 2001, 105, 328.

(35) Lobo, M. M. Electrolyte Solutions: Literature data on thermodynamic and transport properties; Coimbra, 1975.

(36) Paduano, L.; Sartorio, R.; D’Errico, G.; Vitagliano, V. J. Chem. Soc., Faraday Trans. 1998, 94, 2571.

(37) Hinton, J. F.; Amis, E. S. Chem. Rev. 1971, 71, 627.

(38) Millero, F. J. Chem. Rev. 1971, 71, 147.

(39) Vitagliano V.; Sartorio R.; Scala S.; Spaduzzi D. J. Solution Chem. 1978, 7, 605 .

(40) Miller, D. G. In Transport Phenomena in Fluids; Hanley, H. J. M., Ed.; Marcel Dekker: New York, 1969; Chapter 11, p 377.

(41) Ninni, L.; Camargo, M. S.; Meirelles, A. J. A. Thermochim. Acta 1999, 328, 169

(42) Vergara, A.; Paduano, L.; Vitagliano, V.; Sartorio, R. J. Phys. Chem. B 2000, 104 (33), 8086.

(43) Timasheff, S. N. Biochemistry 2002, 41 (46), 13473.

(44) Miller, D. G.; Vitagliano, V.; Sartorio, R. J. Phys. Chem. 1986, 90,1509 .

(45) Akeley, D. K.; Gosting, L. J. J. Phys. Chem. 1953, 20, 5685.

(46) Miller, D. G. J. Phys. Chem. 1988, 92, 4222.

(47) Miller, D. G. J. Phys. Chem. 1981, 85, 1137.

(48) Miller, D. G.; Ting, A. W.; Rard, J. A. J. Electrochem. Soc. 1988 35,896 .

(49) Rard, J. A.; Miller, D. G. J. Phys. Chem. 1988, 92, 6133.

(50) Rard, J. A.; Miller, D. G. J. Solution Chem. 1990, 19, 129. 